BULL. AUSTRAL. MATH. SOC.

VOL. $24(1981), 357-366$.

\title{
INVEX FUNCTIONS AND \\ CONSTRAINED LOCAL MINIMA
}

\author{
B.D. Craven
}

If a certain weakening of convexity holds for the objective and all constraint functions in a nonconvex constrained minimization problem, Hanson showed that the Kuhn-Tucker necessary conditions are sufficient for a minimum. This property is now generalized to a property, called $K$-invex, of a vector function in relation to a convex cone $K$. Necessary conditions and sufficient conditions are obtained for a function $f$ to be $K$-invex. This leads to a new second order sufficient condition for a constrained minimum.

\section{Introduction}

A real function $f: \mathbb{R}^{n} \rightarrow \mathbb{R}$ will be called invex, with respect to $n$, if for the function $n: \mathbb{R}^{n} \times \mathbf{R}^{n} \rightarrow \mathbb{R}^{n}$,

$$
f(x)-f(u) \geq f^{\prime}(u) n(x, u)
$$

holds for each $x$ and $u$ in the domain of $f$. Here $f^{\prime}(u)$ is the Fréchet derivative of $f$ at $u$. Hanson [5] (see also [6], [7]) introduced this concept, and showed that, if all the functions $f_{i}$ in the (nonconvex) constrained minimization problem,

(2) Minimize $f_{0}(x)$ subject to $f_{i}(x) \leq 0 \quad(i=1,2, \ldots, m)$, are invex, with respect to the same $n$, then the Kuhn-Tucker conditions

Received 27 July 1981. 
necessary for a global minimum of (2) are also sufficient. In fact, Hanson's proof [5] does not require ( 1 ) at all points $x$ and $u$, since $u$ may be fixed at the point where the Kuhn-Tucker conditions hold. Define, therefore, a real function $f$ to be invex in a neighbourhood at $u$ if $f$ satisfies (1) for a given $u$, and for all $x$ such that $\|x-u\|$ is sufficiently small. With this definition, the Kuhn-Tucker necessary conditions become also sufficient for a local minimum; the proof is the same as Hanson's. Craven [2] has shown that $f$ has the invex property when $f=h \circ \phi$, with $h$ convex, $\phi$ differentiable, and $\phi^{\prime}$ having full rank. Thus some invex functions, at least, may be obtained from convex functions by a suitable transformation of the domain space. Such transformations destroy convexity, but not the invex property; the term invex, from invariant convex, was introduced in [2] to express this fact. (In (1), $f$ is convex if $\eta(x, u)=x-u$.)

The requirement that all the functions $f_{i}$ in (2) are invex with respect to the same function $\eta$ may be expressed by forming a vector $f$, whose components are $f_{i}(i=0,1,2, \ldots, m)$, and then requiring that

$$
f(x)-f(u)-f^{\prime}(u) \eta(x, u) \in \mathbb{R}_{+}^{m+1},
$$

where $\mathbb{R}_{+}^{m+1}$ denotes the nonnegative orthant in $\mathbb{R}^{m+1}$. More generally, let $K \subset \mathbf{R}^{m+1}$ be a convex cone. The vector function $f: \mathbf{R}^{n} \rightarrow \mathbf{R}^{m+1}$ will be called $K$-invex, with respect to $\eta$, if

$$
f(x)-f(u)-f^{\prime}(u) n(x, u) \in K
$$

for all $x$ and $u$. If $u$ is fixed, and (4) holds whenever $\|x-u\|$ is sufficiently small, then $f$ will be called $k$-invex, with respect to $\eta$, in a neighbourhood at $u$. It is noted that, if $f$ is $K$-invex in a neighbourhood at $u$, and if $v \in K^{*}$ (the dual cone of $K$, thus $\left.v(K) \subset \mathrm{R}_{+} \equiv[0, \infty)\right)$, then $v^{T} f$ is invex in a neighbourhood at $u$, with respect to the same $n$.

In this paper, conditions are obtained necessary, or sufficient, for $f$ to be $K$-invex with respect to some $n$. This involves an investigation of appropriate functions $\eta$ for (4). To motivate the generalization to cones, consider problem (2) generalized to 
Minimize $f_{0}(x)$ subject to $-g(x) \in S$,

where $g(x)=\left(f_{1}(x), f_{2}(x), \ldots, f_{m}(x)\right)^{T}$, and $s \subset \mathbb{R}^{m}$ is a convex cone. The Kuhn-Tucker conditions necessary (assuming a constraint qualification) for a minimum of (5) at $x=u$ are that a Lagrange multiplier $\theta \in S^{*}$ exists, for which

$$
f_{0}^{\prime}(u)+\theta^{T} g^{\prime}(u)=0 ; \quad \theta^{T} g(u)=0 .
$$

Set $\lambda=(1, \theta)$, and $K=\mathbb{R}_{+} \times S^{*}$. (If $S=\mathbb{R}_{+}^{m}$ then $K=\mathbb{R}_{+}^{m+1}$.) Then (6) may be rewritten as

$$
\lambda^{T} f^{\prime}(u)=0 ; \quad \lambda^{T} f(u)=f_{0}(u) ; \lambda \in K^{*}
$$

The following converse Kuhn-Iucker theorem then holds.

THEOREM 1. Let $u$ be feasible for problem (5); let the Kuhn-Tucker conditions (7) hold at $u$, with $\lambda_{0}=1$; let $f$ be $K$-invex, with respect to some $\eta$, in a neighbourhood at $u$. Then $u$ is a local minimon of (5).

Proof. Let $x$ be any feasible point for (5), with $\|x-u\|$ sufficiently small. Then

$$
\begin{aligned}
f_{0}(x)-f_{0}(u) & \geq \lambda^{T} f(x)-\lambda^{T} f(u) \text { since } \theta^{T} g(x) \leq 0 \text { and } \lambda^{T} f(u)=f_{0}(u) \\
& \geq \lambda^{T} f^{\prime}(u) \eta(x, u) \text { by the invex hypothesis } \\
& =0 \text { by the Kuhn-Tucker conditions. }
\end{aligned}
$$

So $u$ is a local minimum for (5).

The result generalizes [7], Theorem 2, which applies to polyhedral cones $S$ only.

If the problem (5) is not convex, then the hypotheses (and proof) of Theorem 1 lead to a local (but not necessarily global) minimum. A local minimum also follows if $f$ is $U$-invex, where $U$ is a convex cone containing $K$, and $\lambda \in U^{*}$. Here the vector function $f$ is less restricted than in Theorem 1 , and the Lagrange multiplier $\lambda$ is more restricted.

The problem (2) is equivalent to the transformed problem, 
(8) Minimize $\phi_{0} \circ f_{0}(x)$ subject to $\phi_{i} \circ f_{i}(x) \leq 0 \quad(i=1,2, \ldots, m)$, in the sense that both problems (2) and (8) have the same feasible set and the same minimum (local or global), provided that $\phi_{0}: \mathbb{R} \rightarrow \mathbb{R}$ is strictly increasing and, for $i=1,2, \ldots, m, \phi_{i}\left(\mathbb{R}_{+}\right) \subset \mathbf{R}_{+}$and $\phi_{i}\left(-\mathbb{R}_{+}\right) \subset \mathbb{R}_{+}$. (The $\phi_{i}$, for $i=1,2, \ldots, m$, may be monotone, but need not be so.) Let $F$ denote the vector whose components are $\phi_{0} \circ f_{0}, \phi_{1} \circ f_{1}, \ldots, \phi_{m} \circ f_{m}$. The converse Kuhn-Tucker property will hold for the original problem (2) if it holds for the transformed problem (8), thus if $F$ is $R_{+}^{m+1}$-invex, with respect to some $n$. Although (8) is not generally a convex problem, a local minimum for ( 8 ) at $u$ follows, as in Theorem 1; and this implies a local (and hence global) minimum at $u$ for the convex problem (2).

\section{Conditions necessary or sufficient for an invex function}

Assume now that the vector functions $f$ and $n$ are twice continuously differentiable. For fixed $u$, the Taylor expansion of $n(x, u)$ in terms of $v=x-u$ gives, up to quadratic terms

$$
n(x, u)=\eta_{0}+A v+\frac{1}{2} v^{T} Q_{0} v+o\left(\|v\|^{2}\right) \quad(v=x-u),
$$

where $A$ is an $n \times n$ matrix of first partial derivatives, and $v^{T} Q . v$ is a coordinate-free notation (see [3]) for the vector whose $k$ th component is

$$
\sum_{i, j=1}^{n} v_{i} Q_{k, i j} v_{j} \text {, where } Q_{k, i j}=\left.\frac{\partial^{2} \eta_{k}(x, u)}{\partial x_{i} \partial x_{j}}\right|_{x=u}
$$

Of course, $\eta_{0} \equiv \eta(u, u), A$, and $Q$. depend on $u$. If $q$ is a row vector with $n$ components, let $q Q$. denote the matrix $Q_{k}$ whose elements are $\sum_{k=1}^{n} q_{k} Q_{k, i j}$. Thus $q\left(v^{T} Q_{.} v\right)=v^{T}\left(q Q_{.}\right)_{\nu}$, an ordinary quadratic form. Similarly, $f$ has an expansion

$$
f(x)-f(u)=B v+\frac{1}{2} v^{T} M v+o\left(\|v\|^{2}\right),
$$


where $B=f^{\prime}(u)$ is a matrix of first derivatives $\partial f_{k}(x) /\left.\partial x_{i}\right|_{x=u}$, and

$$
M_{k, i j}=\left.\frac{\partial^{2} f_{k}(x)}{\partial x_{i} \partial x_{j}}\right|_{x=u}
$$

Let $S$ be a convex cone in $\mathbb{R}^{n}$. The quadratic expression $v^{T} M_{\bullet} v$ will be called S-positive semidefinite if $v^{T} M, v \in S$ for every $v \in \mathbb{R}^{n}$. The expression $v^{T} M . v$ will be called s-positive definite if $v^{T} M . v \in$ int $S$ for every nonzero $v \in \mathbb{R}^{n}$. Here int $S$ denotes the interior of $S$, supposed nonempty. Now $v^{T} Q_{k} v$ can be expressed, by rotation of axes, in the form $\sum_{i=1}^{n} \rho_{k i} \alpha_{k i}$, where the $\rho_{k i}$ $(i=1,2, \ldots, n)$ are the eigenvalues of $Q_{k}$, and the $\alpha_{k i}$, depending on $v$, are nonnegative. For each $k$, denote by $\rho_{k}$ the vector whose components are $\rho_{k I}, \rho_{k 2}, \ldots, \rho_{k n}$. If, for some ordering of the eigenvalues of each $Q_{k}$, every vector $\rho_{k}$ lies in $S$ (respectively in int $S$ ), then it follows that $v^{T} Q . v$ is $S$-positive semidefinite (respectively $S$-positive definite). This sufficient condition for $S$-positive semidefiniteness would be also necessary if the $Q_{k}$ are simultaneously diagonalizable, but that is not usually the case. It is convenient to say that $Q$. is S-positive (semi-) definite when $v^{T} Q . v$ is.

If $S$ is a polyhedral cone, then the dual cone $S^{*}$ has a finite set, $G$, of generators (considered as row vectors). Since a vector $a \in S$ if and only if $q s \geq 0$ for each $q \in G$, it follows that $Q$ is $S$-positive (semi-) definite if and only if, for each $q \in G, q Q$ is positive (semi-) definite in the usual sense.

Let $r=m+1$. If $B$ is an $r \times n$ matrix, define $v^{T}(B Q)$.$v for$ $v \in \mathbb{R}^{n}$ as the vector whose $k$ th component is 


$$
\sum_{i, j=1}^{n} v_{i} C_{k, i j} v_{j} \text { where } C_{k, i j}=\sum_{t=1}^{r} B_{k t^{Q} t, i j} .
$$

Let I denote the $n \times n$ identity matrix.

Observe that the $K$-invex property $(4)$ is unaffected by subtracting from $\eta$ any term in the nullspace of $f^{\prime}(u) \equiv B$.

THEOREM 2. Let $f: \mathbb{R}^{n} \rightarrow \mathbb{R}^{r}$ be twice continuously differentiable: Let $K \subset \mathbb{R}^{r}$ be a closed convex cone, satisfying $K \cap(-K)=\{0\}$. If $f$ is $k$-invex in a neighbourhood at $u$, with respect to a twice continuously differentiable function $n$, for which $n(u, u)=0$, then, after subtraction of a term in the nullspace of $B, \eta$ has the form

$$
n(u+v, u)=v+\frac{1}{2} v^{T} Q_{0} v+o\left(\|v\|^{2}\right),
$$

where $M_{.}-B Q$. is K-positive semidefinite. Conversely, if $\eta$ has the form (14), and if $M .-B Q$. is K-positive definite, then $f$ is K-invex in a neighbourhood at $u$, with respect to this $n$.

Proof. Let $f$ be $K$-invex with respest to $\eta$ in a neighbourhood at $u$. Substituting the expansion (9) into the expansion (11), and setting $n_{0}=0$, the inequality

$$
\left[B v+\frac{1}{2} v^{T} M \cdot v+O\left(\|v\|^{2}\right)\right]-B\left[A v+\frac{1}{2} v^{T} Q_{\bullet} v+O\left(\|v\|^{2}\right)\right] \in K
$$

must hold, whenever $\|v\|$ is sufficiently small. Considering the terms linear in $v, B v-B A v+o(\|v\|) \in K$ for each $v \in \mathbb{R}^{n}$. Hence, for each $q \in K^{*}$, and each $v, q(B(I-A) v+o(\|v\|)) \geq 0$, hence $q B(I-A) v \geq 0$. Hence $B(I-A) v \in K \cap(-K)=\{0\}$. Therefore $B(I-A)=0$. But the definition (4) of $K$-invex allows any term in the nullspace of $B$ to be added to $\eta$. Hence $f$ is also $K$-invex with respect to $\eta$, now modified by replacing $A$ by $I$. The quadratic terms then require that, for each $v$,

$$
v^{T}\left(M_{-}-B Q .\right) v+o\left(\|v\|^{2}\right) \in K .
$$

Hence, for each $q \in K^{*}$ and each $\alpha>0$, replacing $v$ by $\alpha v$,

$$
q\left[v^{T}\left(M_{0}-B Q_{0}\right) v\right]+o\left(\alpha^{2}\right) / \alpha^{2} \geq 0 \text {. }
$$


Hence $q\left[v^{T}\left(M_{.}-B_{0}\right) v\right] \geq 0$ for each $q \in K^{*}$, hence

$$
v^{T}\left(M_{.}-B Q .\right) v \in K \text { for each } v \in \mathbf{R}^{n} \text {. }
$$

Thus $M_{0}-B Q$. is $K$-positive semidefinite.

Conversely, assume that $M_{0}-B Q$. is $K$-positive definite. A reversal of the above argument shows that (15), with $A=I$, is satisfied up to quadratic terms, with $K$ replaced by int $K$. Here the quadratic terms dominate any higher order terms, so that (15) itself holds, whenever $\|v\|$ is sufficiently small. Thus (4) follows, and $f$ is $K$-invex, in a neighbourhood at $u$, with $n$ given by (14).

If a nonzero term $n_{0} \equiv(0,0)$ is included in (9), then the $K$-invex property for $f$ requires that $-B n_{0} \in K$, on setting $v=0$. Suppose that, for the constrained minimization problem (5), the Kuhn-Tucker conditions (6) hold at the point $u$. Then $\lambda^{T} B=0$, for some nonzero $\lambda \in K^{*}$. Suppose that $\lambda \in$ int $K^{*}$ (for problem (2), this means that each Lagrange multiplier $\left.\lambda_{i}>0\right)$. From this, if $0 \neq-B n_{0} \in K$, there follows (see [1], page 31) $\lambda^{T} B \dot{1}_{0}<0$, contradicting $\lambda^{T} B=0$. So the assumption that $B n_{0}=0$ is a relevant one, when the $K$-invex property is to be applied to Kuhn-Tucker conditions and Theorem 1 . In Theorem 2, $n_{0}=0$ was assumed, since a vector in the nullspace of $B$ may be subtracted from $n$.

In Theorem 2, the sufficient conditions for $f$ to be $K$-invex involve first and second derivatives of $f$. Combining this with the sufficient Kuhn-Tucker theorem (Theorem 1), it has been shown that the Kuhn-Tucker conditions are sufficient for a local minimum of a nonconvex problem, if the first and second derivatives of $f$ at the Kuhn-Tucker point $u$ are suitably restricted. The Lagrangian $f_{0}(x)+\lambda^{T} g(x)$ for the problem (5) has $\lambda^{T} M$ as its matrix of second derivatives. The second order sufficiency conditions, given by Fiacco and McCormick [4], page 30, require that (in the present notation) each component of $v^{T}\left(\lambda^{T} M_{.}\right) v>0$ for each nonzero $v$ in a certain cone. This is related to, but not the same as, 
the hypothesis (from Theorem 2) that $M .-B Q$. is $K$-positive definite, for some choice of $Q$. . However, the construction of a suitable $Q$, given $f$, is a nontrivial matter, since the eigenvalues of each $M_{k}-(B Q .)_{k}$ matrix are involved.

\section{Examples}

Consider the problem,

$$
\begin{array}{ll}
f_{0}(x)=\frac{1}{3} x_{1}^{3}-x_{2}^{2} \text { subject to } \\
x=\left(x_{1}, x_{2}\right) \in R^{2} \\
& f_{1}(x)=\frac{1}{2} x_{1}^{2}+x_{2}^{2}-1 \leq 0 .
\end{array}
$$

This problem has a local minimum at $(0,1)$, with Lagrange multiplier 1 . The matrices $M_{k}$ are then

$$
M_{0}=\left[\begin{array}{cc}
0 & 0 \\
0 & -2
\end{array}\right] \text { and } M_{1}=\left[\begin{array}{ll}
1 & 0 \\
0 & 2
\end{array}\right] ; \quad B=\left[\begin{array}{cc}
0 & -2 \\
0 & 2
\end{array}\right] \text {. }
$$

When do symmetric matrices $Q_{0}$ and $Q_{1}$ exist for which

$$
\left[\begin{array}{cc}
0 & 0 \\
0 & -2
\end{array}\right]-O Q_{0}-(-2) Q_{1} \text { and }\left[\begin{array}{ll}
1 & 0 \\
0 & 2
\end{array}\right]-O Q_{0}-2 Q_{1}
$$

are both positive semidefinite, or both positive definite? Setting

$$
Q_{1}=\left[\begin{array}{ll}
\alpha & \beta \\
\beta & \gamma
\end{array}\right] \text {, the two matrices are }\left[\begin{array}{cc}
2 \alpha & 2 \beta \\
2 \beta & -2+2 \gamma
\end{array}\right] \text { and }\left[\begin{array}{cc}
1-2 \alpha & -2 \beta \\
-2 \beta & 2-2 \gamma
\end{array}\right] \text {. }
$$

Using the Routh-Hurwicz criterion, $0 \leq \alpha \leq 1, \gamma=1$, $\alpha(-1+\gamma)-\beta^{2} \geq 0$, and $(1-\alpha)(2-2 \gamma)-4 \beta^{2} \geq 0$, are required. Positive semidefinite matrices

$$
\left[\begin{array}{ll}
\frac{1}{2} & 0 \\
0 & 0
\end{array}\right] \text { and }\left[\begin{array}{ll}
\frac{1}{2} & 0 \\
0 & 0
\end{array}\right]
$$

are obtained, with $\alpha=\frac{1}{2}, \beta=0, \gamma=1$, but positive definite matrices are not possible. Thus the necessary conditions of Theorem 2, but not the sufficient condition, holds in this instance. 
For the same problem ( 18$)$, the point $\left(-1,2^{\frac{1}{2}}\right)$ is a saddle point, with Lagrange multiplier 1 . Here

$$
M_{0}=\left[\begin{array}{cc}
-1 & 0 \\
0 & -2
\end{array}\right], M_{1}=\left[\begin{array}{ll}
1 & 0 \\
0 & 2
\end{array}\right], \quad B=\left[\begin{array}{cc}
1 & -2^{\frac{\pi}{2}} \\
-1 & 2^{\frac{1}{2}}
\end{array}\right] \text {. }
$$

The matrices to consider are

$$
\left[\begin{array}{cc}
-1 & 0 \\
0 & -2
\end{array}\right]+Q_{0}-2^{\frac{1}{2} Q_{1}} \text { and }\left[\begin{array}{ll}
1 & 0 \\
0 & 2
\end{array}\right]-Q_{0}+2^{\frac{1}{2} Q_{1}} \text {, }
$$

and these cannot both be positive definite (or semidefinite), whatever the choice of the matrix $Q_{0}-2^{\frac{1}{2}} Q_{1}$. So the sufficient condition of Theorem 2 does not hold here.

\section{References}

[1] B.D. Craven, Mathematical programing and control theory (Chapman and Hall, London; John Wiley \& Sons, New York; 1978).

[2] B.D. Craven, "Duality for generalized convex fractional programs", Generalized concavity in optimization and economies (Academic Press, New York, London, to appear).

[3] B.D. Craven and B. Mond, "Sufficient Fritz John optimality conditions for nondifferentiable convex programming", J. Austral. Math. Soc. Ser. B $19(1975 / 76), 462-468$.

[4] Anthony V. Fiacco, Garth P. McCormick, Nonlinear progromming:

sequential unconstrained minimization techniques (John Wiley and Sons, New York, London, Sydney, 1968).

[5] Morgan A. Hanson, "On sufficiency of the Kuhn-Tucker conditions", $J$. Math. Anal. Appl. 80 (1981), 545-550.

[6] M.A. Hanson and B. Mond, "Further generalizations of convexity in mathematical programming" (Pure Mathematics Research Paper No. 80-6, Department of Mathematics, La Trobe University, Melbourne, 1980). See also: J. Inform. Optim. Sci. (to appear). 
[7] B. Mond and M.A. Hanson, "On duality with generalized convexity" (Pure Mathematical Research Paper No. 80-4, Department of Mathematics, La Trobe University, Melbourne, 1980).

Department of Mathematics, University of Melbourne, Parkville, Victoria 3052, Australia. 